\title{
EFFECTIVENESS OF VISUAL COLLISION WARNING ALERTS ON YOUNG DRIVERS' LATENT HAZARD ANTICIPATION
}

\author{
Foroogh Hajiseyedjavadi ${ }^{1}$, Ravi Agrawal ${ }^{1}$, Donald Fisher ${ }^{2} \&$ Siby Samuel $^{1}$ \\ ${ }^{1}$ University of Massachusetts Amherst, ${ }^{2}$ Volpe National Transportation Center \\ Email: f.hajiseyedjavadi@gmail.com
}

\begin{abstract}
Summary: Forward roadway collision warning systems can reduce rear-end collisions, among other unsafe behaviors. Previous studies have shown that young drivers fail to scan adequately for latent hazards. The current driving simulator study investigates the effect of visual collision warning messages on drivers' hazard anticipation ability, when presented either $2 \mathrm{~s}, 3 \mathrm{~s}$ or $4 \mathrm{~s}$ in advance of a potential threat. This experiment examined the latent hazard anticipation behavior of forty-eight young drivers aged 18-25 across eight unique scenarios both, in the presence, and absence of visual collision warning alerts. The analysis of glance data captured using an eye tracker, show that visual warning messages significantly increased the proportion of latent hazards anticipated regardless of hazard type (pedestrian or vehicle). The $2 \mathrm{~s}$ warning duration was found to statistically have the same effect on hazard anticipation compared to the $3 \mathrm{~s}$ and $4 \mathrm{~s}$ warning thresholds. The study has potential implications for the effective design of forward collision warning systems.
\end{abstract}

\section{INTRODUCTION}

Rear end collisions account for approximately $28 \%$ of all collisions and cause approximately 157 million vehicle-hours of delay (Parasuraman, Hancock \& Olofinboba 1997). Forward roadway collision systems can aid drivers with reducing their involvement in rear-end crashes (Maltz, Shinar 2004). As defined by the Society of Automotive Engineers (SAE), collision avoidance systems fall under the category of Level 1 or function-specific lower level automation (Blanco et al. 2015, Smith 2013). Typical lane departure collision avoidance systems are lateral control systems that provided warnings and control assistance to the driver merely via lane tracking (Parasuraman, Hancock \& Olofinboba 1997). More sophisticated systems include speed and steering control for more significant impacts on the reduction of crashes (Mazzae, Garrott \& Flick 1995), while intersection collision warning systems specifically aim to improve the situation awareness of drivers by appropriately notifying them via visual and audio messages, of potential dangerous situations that may lead to a collision (Miller \& Huang 2002, Fung et al. 2007). Several studies have investigated the effectiveness of collision warning alerts. One such simulator study examining the effectiveness of audio alerts for an intersection collision warning system showed that drivers receiving the auditory alert had a shorter reaction time, lower speed and reduced crash rate compared to drivers receiving no such alerts (Chang et al. 2009). In a distracted driving context, a simulator study by Lee showed that graded warnings provided a greater safety margin and elicited fewer inappropriate responses to poor alarms from drivers (Lee, Hoffman \& Hayes 2004). Another simulator study showed that audiovisual collision avoidance warnings elicited the best performance in terms of alerting drivers to potential forward and side object collisions, and older drivers were found to benefit as much as younger drivers from such alerts (Kramer et al. 2007). However, none of these studies have explicitly determined the effectiveness of such alerts on improving drivers' anticipation behavior in the presence of potential threats. Latent hazard anticipation is defined as the ability to appropriately scan visual areas of the roadway that contain a latent hazard, materialized or otherwise (e.g. a pedestrian attempting to cross occluded by a truck). Multiple studies showed that novice drivers fail more 
frequently to perceive hazardous locations in the roadway and perform appropriate scanning behaviors accordingly compared to middle-aged drivers (Pradhan et. al. 2005, Chan et. al. 2010). Collision warning systems, might benefit teen drivers by compensating for their lack of hazard anticipation and attention maintenance skills. While the modality of the alert is important for its effectiveness, the timing of the warnings is equally critical. Poorly timed warnings may undermine the driver's safety (McGehee et al. 2002). An early alert may be ignored by the drivers while, late alerts may disrupt a concurrent vehicular maneuver (Lee et al. 2002). A simulator assessment of adaptive collision warning systems showed that mistimed alerts lead to diminished trust and therefore, inappropriate responses from the driver (Jamson, Lai \& Carsten 2008). Though literature broadly examines various alert timings for effectively warning drivers of impending collisions, no reported study has determined the timing threshold for these warnings to improve young drivers' latent hazard anticipation ability to the levels observed for experienced drivers.

\section{OBJECTIVES}

The current research both, examines the effectiveness of visual alerts at improving drivers' latent hazard anticipation behavior, and assesses an effective threshold for the effective alerts, in a context where drivers are fully attentive and paying attention to the forward roadway. There are 2 related hypotheses: First, the effect of timing of the visual collision warning alert on latent hazard anticipation is evaluated. Three different alert thresholds $(2 \mathrm{~s}, 3 \mathrm{~s}$ and $4 \mathrm{~s})$ are examined and compared with a control condition of drivers that receive no such alert. Among the three warning thresholds studied in this paper, drivers are expected to anticipate a greater proportion of hazards in the warning condition with the longest warning threshold ( $4 \mathrm{~s}$ in this study). Second, the effecttiveness of the visual alerts at differentially improving anticipation behaviors for different hazard types (pedestrians and vehicles) is assessed. Drivers are expected to be less likely to detect a pedestrian latent hazard compared to a vehicle hazard, since vehicle hazards are most of the times behavioral prediction (BP) hazards while pedestrian hazards are more often environmental prediction (EP) hazards (Crundall et. al. 2012).

\section{METHODOLOGY}

In the current between subjects design, forty-eight participants ( 24 female and 24 male) aged 1825 years, with an average age of 20.5 years $(S D=2.3)$ and, an average driving experience of 3.3 years ( $\mathrm{SD}=2.2$ ) completed the experiment. All participants were recruited from the University of Massachusetts Amherst and local neighborhood and were remunerated for their participation.

\section{Apparatus}

A driving simulator and an eye tracker were used in the current experiment. The driving simulator used in the current study is a Realtime Technologies Inc. (RTI) full cab, fixed base Saturn sedan with three screens (equipped with overhead projectors) that subtend 150 degrees of horizontal field of view and 30 degrees' vertical field of view. The simulator is equipped with a surround sound system that generates appropriate environment, and Doppler effects in addition to the availability of complete vehicle controls for navigation of the virtual environment. An Applied Science Laboratories (ASL) Mobile Eye, head mounted eye tracking system was used to track and record drivers' eye movements during the experiment. The eye tracker recording frequency is 30 frames per second and its accuracy is 0.5 degrees of visual angle. 


\section{Scenarios \& Visual Warnings}

The experimental design included 8 different scenarios (with one hazard each) administered across 4 drives of 2 scenarios each. The ordering of the drives was completely counterbalanced both, across and within groups. There were four groups of drivers in this between subjects design. Drivers either navigated virtual scenarios with visual collision warning messages presented 2, 3, or 4 seconds before the hazard for the alerting conditions, or with no such messages for the control group (four groups: $2 \mathrm{~s}, 3 \mathrm{~s}, 4 \mathrm{~s}$ and control group). As an example, for one of the pedestrian scenarios there is a mid-block crosswalk on subject's path having some bushes covered each end of the crosswalk so that the presence of a potential pedestrian is uncertain to the driver, and a pedestrian crossing sign is placed upstream of the roadway. The scenarios and latent hazards have been described in full and previously validated in other studies (Samuel et al. 2016). The visual warning alerts developed and evaluated in the current study are presented in.

Table 1. Visual warning alerts

\begin{tabular}{|c|c|c|c|c|c|c|}
\hline $\begin{array}{c}\text { Pedestrian } \\
\text { Visual } \\
\text { Warnings }\end{array}$ & & & & & & \\
\hline
\end{tabular}

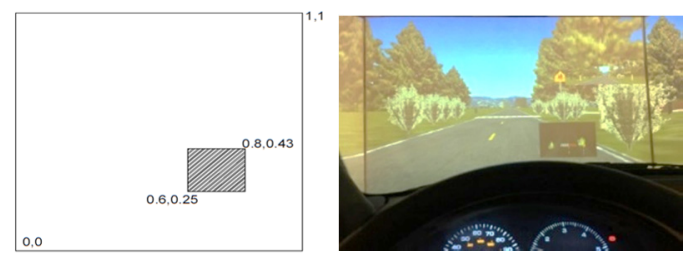

Figure 1. The layout of visual warning alert (with respect to the center screen of the simulator)

The visual collision warning messages were animated messages presenting during the simulated driving task (Table 1) with the potential hazard highlighted and repeatedly flickering (red color), and an indication for the direction of the hazards' movement provided in some cases (3 cases). The visual warning alerts were presented on the center screen of the simulator towards the bottom right corner (see Figure 1 for orientation). Left panel of Figure 1 shows a geometric view of where the warnings were presented, relative to the center screen of the simulator while the right panel of the figure shows a perspective view of one virtual scenario on the center channel, with the corresponding warning displayed in advance of a potential pedestrian threat.

\section{Experimental Design \& Procedure}

A between subjects' design was employed where each subject completed all the 4 drives (with 2 scenarios) once, with either one of the collision warning alert conditions $(2,3$, or $4 \mathrm{sec})$ or as the control group. There were four groups in total and participants were pseudo-randomly assigned to one of the groups. The sequence of drives presented to the subjects was completely counterbalanced using a Latin square method (Winer, Brown \& Michels, 1971), across drives. Partici- 
pants provided written informed consent to participate in the experiment. The participants then completed a single questionnaire related to their driving history and demographic information. Following this, participants were outfitted with an eye tracker and their eyes were calibrated. Supplementary instructions were provided to participants at the onset of each drive. A practice drive was provided to all subjects to familiarize them with the controls of the simulator and the simulated environment. Finally, the participants were asked to navigate the experimental scenarios either with, or without warning. Participants were asked to maintain a speed limit of 35 miles per hour throughout all scenarios in the experiment. The complete experiment averaged 35 minutes in total duration.

\section{Dependent Variables}

The dependent variable in the current study is the drivers' hazard anticipation ability measured by whether the driver glanced at the hazard or not. The dependent variable is scored 1, if the driver appropriately glances at the pre-determined target zone while in the launch zone (and 0 otherwise). As defines in previous studies, the target zone here is defined as the area of the roadway that the driver should scan to anticipate a potential hazard. The launch zone is defined as that area of the roadway where the driver should begin glancing at the target zone to be able to successfully anticipate and mitigate the threat (Samuel, Fisher 2015).

\section{RESULTS}

The dependent variable in this study was aggregated across each participant. Every subject in this experiment drove attentively without any distraction throughout the session. The data was binary coded and therefore binomially distributed. To analyze the proportion of latent hazards anticipated across the various visual warning durations, a logistic regression model within the Generalized Estimation Equations (GEE) model was used throughout for all analyses. Participants were added as a random effect in the model, and there was one between subject factor-(a) Treatment: Control or one of the warning conditions ( 2 seconds, 3 seconds, or 4 seconds) - and one within subject factor--(b) Scenario (as described in the methods). A backwards elimination procedure was used to examine the main effect and wean all interaction effects.

\section{Proportion of Pedestrian Latent Hazard Anticipation}

Drivers with visual warning messages presented to them 2 seconds before a hazard, anticipated a lower proportion of the pedestrian threats $(89 \%)$ compared to drivers in the $3 \mathrm{~s}(92 \%)$ and $4 \mathrm{~s}$ (91\%) conditions. Drivers in the control group only anticipated $75 \%$ of the pedestrian threats. The logistic regression model used to analyze the proportion of pedestrian latent hazards anticipated across the various scenarios, presented a highly significant main effect for both Treatment and Scenario [Wald $\chi_{2}^{2}=17.26 ; \mathrm{p}<0.0002$ ].

Also, a set of t-tests was used to compare the percentage of pedestrian latent hazard anticipation across the 4 groups (the 4 seconds, 3 seconds, 2 seconds visual warning groups, and the control group). The results showed that the drivers in all three warning groups anticipated a statistically greater proportion of the latent hazards compared to the control group ( $2 \mathrm{~s}$ vs control: $[\mathrm{t}=3.592$, $\mathrm{p}<0.0005]$, 3s vs control [ $\mathrm{t}=3.834, \mathrm{p}<0.0002]$, and $4 \mathrm{~s}$ vs control: $[\mathrm{t}=3.583, \mathrm{p}<0.0006])$. However, the set of pairwise t-test comparisons between the warning groups did not show any statistical difference for the proportion of latent hazards anticipated across any of the warnings groups, 
implying that the proportion of pedestrian latent hazards anticipated is statistically similar across the $2 \mathrm{~s}, 3 \mathrm{~s}$ and $4 \mathrm{~s}$ thresholds.

\section{Proportion of Vehicle Latent Hazard Anticipation}

The proportion of vehicle latent threats anticipated across all scenarios involving a vehicle hazard was descriptively lower for the 2 seconds warning group (94\%) compared to the 3s (97\%) and $4 \mathrm{~s}(97 \%)$ warning groups. The control group participants anticipated only $69 \%$ of the vehicle threats. The logistic regression model used to analyze the proportion of vehicle latent hazards anticipated across the various visual warning durations, presented a highly significant main effect only for Treatment [Wald $\left.\chi_{2}^{2}=32.81 ; \mathrm{p}<.0001\right]$. A set of t-tests was used to compare the percentage of vehicle latent hazards anticipated across the 4 groups ( 2 seconds, 3 seconds, 4 seconds visual warning groups, and the control group). The results showed that the participants in all the three warning groups exhibited statistically higher anticipation rates compared to the control group ( $2 \mathrm{~s}$ vs control: [ $\mathrm{t}=2.697, \mathrm{p}<0.01$ ], $3 \mathrm{~s}$ vs control: [ $\mathrm{t}=3.003, \mathrm{p}<0.004]$, and $4 \mathrm{~s}$ vs control: [ $\mathrm{t}=3.245, \mathrm{p}<0.002]$ ). The set of pairwise $\mathrm{t}$-test comparisons between the warning groups did not indicate any statistical difference for hazard anticipation rates across any of the warnings groups, implying that the proportion of vehicle latent threats anticipated was similar across the $2 \mathrm{~s}, 3 \mathrm{~s}$ and $4 \mathrm{~s}$ warning thresholds.

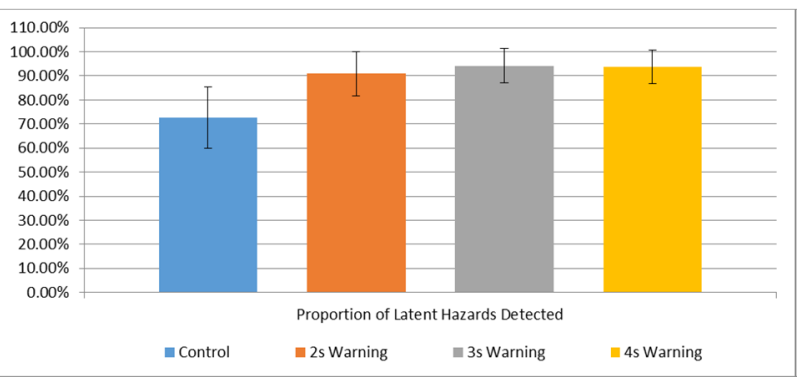

Figure 2. Proportion of latent hazards anticipated

\section{Final Model Including Both Pedestrian and Vehicle Latent Hazard Anticipation}

Finally, a general latent hazard anticipation model was implemented, considering both pedestrian and vehicle latent hazard scenarios (see Figure 2), using a logistic regression model within the framework of Generalized Estimation Equations (GEE). The error bars in the figure are standard deviations of each group. In this model, Latent Hazard type (pedestrian, vehicle) was additionally included as a within subject factor. Using a backward elimination method, the final model showed a statistically significant main effect only for Treatment [Wald $\chi_{2}^{2}=19.08 ; \mathrm{p}<.0001$ ]. A set of pairwise t-test comparisons between the three warning groups did not show any statistical difference for hazard detection rate across any of the warnings groups. Thus, the 2 seconds warning has statistically the same effect on latent hazard anticipation as the $3 \mathrm{~s}$ and $4 \mathrm{~s}$ warning thresholds.

\section{DISCUSSION}

The study investigated the effectiveness of the timing of visual warning messages at increasing the proportion of latent hazards anticipated by participant drivers across the various hazard types (pedestrians and vehicles). Previous latent hazard anticipation studies have shown that young drivers fail to scan adequately for latent hazards. As hypothesized, drivers that received visual 
warning messages anticipated greater proportion of latent pedestrian hazards $(89 \%, 92 \%$, and 91\% for 2 seconds, 3 seconds, and 4 seconds warning groups respectively) compared to the drivers that drove the same scenarios without any warning message presented to them (75\%). Similarly drivers that received warnings had higher latent vehicle hazard anticipation $(94 \%$, $97 \%$, and $97 \%$ for 2 seconds, 3 seconds, and 4 seconds warning groups, respectively) compared to the drivers without the visual warning $(69 \%)$. The proportion of latent hazards anticipated in each of the warning groups was higher than the control condition across both, pedestrian and vehicle threats. The results indicate that appropriate visual alerts can improve the latent hazard anticipation ability of young drivers, in the presence of potential threats such as vehicles and pedestrians. However, when comparing the effectiveness of the timing of alerts, the 2-seconds warnings ( $89 \%$ for pedestrian threats vs $94 \%$ for vehicle threats) appeared to be every bit as effective as the $3 \mathrm{~s}$ and $4 \mathrm{~s}$ warning alerts, when it came to improving the proportion of hazards anticipated by the participants. In other words, the visual warning alerts when presented $2 \mathrm{~s}$ in advance of a potential threat significantly improves the anticipation ability of young drivers (Pradhan et al., 2005). The results of this study shed some light on the utility of effective visual alerts to improve the anticipation ability of drivers in the presence of threats that can lead to a rear end collision, such as sudden braking due to late recognition of a pedestrian at a crosswalk, or a failure to notice an obvious change in the cross traffic while navigating an unsignalized intersection, or rotary. The study has several limitations. While the current paper investigated the effect of visual collision warning messages on hazard anticipation behavior, no measure of the effectiveness of the warnings on hazard mitigation behavior was obtained. The ability to respond to a threat following anticipation is important to safety. Other experiments such as the one by Chan, report better effectiveness with alternate modalities such as tactile and mixed modalities and, show that tactile warnings delivered at a $3 \mathrm{~s}$ threshold are more effective than single-mode, visual or auditory alerts (Chan and $\mathrm{Ng}, 2009$ ). In a future study, it would be useful to determine whether the utility of mixed-modal or multi-modal messages can further improve hazard anticipation ability. An on-road study should be undertaken to determine the impact on real world behavior and the assessment of age related effects of such warnings can also shed light on user-centric interface design. The scenario set tested was relatively small. A larger classification of scenarios might show more interesting trends. In summary, the results of this study illustrate the effectiveness of visual alerts as well as the effect of appropriate timing of the alerts on drivers' hazard anticipation, which is critical for designing ADAS systems. There exist potential design implications, pending further research.

\section{ACKNOWLEDGEMENTS}

A Tier 1 award from the Department of Transportation University Center to the University of Iowa supported this research. The opinions expressed herein are solely those of the individual authors.

\section{REFERENCES}

Blanco, M., Atwood, J., Vasquez, H.M., Trimble, T.E., Fitchett, V.L., Radlbeck, J., Fitch, G.M., Russell, S.M., Green, C.A. \& Cullinane, B. 2015, Human factors evaluation of level 2 and level 3 automated driving concepts

Chan, A. H., \& Ng, A. W. 2009, "Perceptions of implied hazard for visual and auditory alerting signals", Safety Science, vol. 47, no. 3, pp. 346-352 
Chan, E., Pradhan, A.K., Pollatsek, A., Knodler, M.A. and Fisher, D.L. 2010, "Are driving simulators effective tools for evaluating novice drivers' hazard anticipation, speed management, and attention maintenance skills?", Transportation research part F: traffic psychology and behaviour, vol. 13, no. 5, pp. 343-353.

Chang, S., Lin, C., Hsu, C., Fung, C. \& Hwang, J. 2009, "The effect of a collision warning system on the driving performance of young drivers at intersections", Transportation research part F: traffic psychology and behaviour, vol. 12, no. 5, pp. 371-380.

Crundall, D., Chapman, P., Trawley, S., Collins, L., Van Loon, E., Andrews, B. and Underwood, G. 2012, "Some hazards are more attractive than others: Drivers of varying experience respond differently to different types of hazard", Accident Analysis \& Prevention, no. 45, pp. 600-609.

Fung, C., Chang, S., Hwang, J., Hsu, C., Chou, W. \& Chang, K. 2007, "The study on the influence of audio warning systems on driving performance using a driving simulator", Proceedings of the 20th International Technical Conference on the Enhanced Safety of Vehicles, Lyon, pp. 18.

Jamson, A.H., Lai, F.C. \& Carsten, O.M. 2008, "Potential benefits of an adaptive forward collision warning system", Transportation Research Part C: Emerging Technologies, vol. 16, no. 4, pp. 471484.

Kramer, A.F., Cassavaugh, N., Horrey, W.J., Becic, E. \& Mayhugh, J.L. 2007, "Influence of age and proximity warning devices on collision avoidance in simulated driving", Human factors, vol. 49, no. 5, pp. 935-949.

Lee, J.D., McGehee, D.V., Brown, T.L. \& Reyes, M.L. 2002, "Collision warning timing, driver distraction, and driver response to imminent rear-end collisions in a high-fidelity driving simulator", Human factors, vol. 44, no. 2, pp. 314-334.

Lee, J.D., Hoffman, J.D. \& Hayes, E. 2004, "Collision warning design to mitigate driver distraction", Proceedings of the SIGCHI Conference on Human factors in Computing SystemsACM, pp. 65.

Maltz, M. \& Shinar, D. 2004, "Imperfect in-vehicle collision avoidance warning systems can aid drivers", Human factors, vol. 46, no. 2, pp. 357-366.

Mazzae, E.N., Garrott, W.R. \& Flick, M.A. 1995, Human factors evaluation of existing side collision avoidance system driver interfaces.

McGehee, D., Brown, T., Lee, J. \& Wilson, T. 2002, "Effect of warning timing on collision avoidance behavior in a stationary lead vehicle scenario", Transportation Research Record: Journal of the Transportation Research Board, no. 1803, pp. 1-6.

McKnight, A.J. and McKnight, A.S. 2003, "Young novice drivers: careless or clueless?", Accident Analysis \& Prevention, vol. 35, no. 6, pp. 921-925.

Miller, R. \& Huang, Q. 2002, "An adaptive peer-to-peer collision warning system", Vehicular technology conference, 2002. VTC Spring 2002. IEEE 55 ${ }^{\text {th }}$ IEEE, pp. 317.

Parasuraman, R., Hancock, P. \& Olofinboba, O. 1997, "Alarm effectiveness in driver-centered collisionwarning systems", Ergonomics, vol. 40, no. 3, pp. 390-399.

Pradhan, A. K., Hammel, K. R., DeRamus, R., Pollatsek, A., Noyce, D. A., \& Fisher, D. L. 2005, "Using eye movements to evaluate effects of driver age on risk perception in a driving simulator", Human Factors: The Journal of the Human Factors and Ergonomics Society, vol 47, no. 4, pp. 840-852.

Pradhan, A.K., Pollatsek, A., Knodler, M. \& Fisher, D.L. 2009, "Can younger drivers be trained to scan for information that will reduce their risk in roadway traffic scenarios that are hard to identify as hazardous?", Ergonomics, vol. 52, no. 6, pp. 657-673.

Samuel, S. \& Fisher, D.L. 2015, "Evaluation of the Minimum Forward Roadway Glance Duration", Transportation Research Record: Journal of the Transportation Research Board, no. 2518, pp. 9-17.

Samuel, S., Borowsky, A., Zilberstein, S. \& Fisher, D.L. 2016, "Minimum Time to Situation Awareness in Scenarios Involving Transfer of Control from Automated Driving Suite", Transportation Research Board 95th Annual Meeting.

Smith, B.W. 2013, "SAE levels of driving automation", Center for Internet and Society. Stanford Law School.http://cyberlaw.stanford.edu/blog/2013/12/sae-levels-drivingautomation. 